\title{
El proceso de Bolonia en el área de contabilidad: Efectos sobre la satisfacción de los estudiantes*
}

\section{The Bologna process in accounting: effects on students satisfaction}

Ester Oliveras (ester.oliveras@upf.edu)

Patrícia Crespo (patricia.crespo@upf.edu)

Josep Maria Raya (josep.raya@upf.edu)

Universitat Pompeu Fabra (España)

http://dx.doi.org/10.12795/EDUCADE.2013.i04.03

RESUMEN: La adaptación de las universidades españolas al Espacio Europeo de Educación Superior (EEES) ha introducido un cambio de modelo educativo. Tradicionalmente, la educación universitaria en España se ha centrado en el marco del Modelo de Instrucción, cuya base es proveer instrucción. Sin embargo, con la introducción experimental al EEES, se observa una tendencia hacia el Modelo de Aprendizaje, cuya base es proveer aprendizaje.

El artículo analiza los efectos sobre la satisfacción de los estudiantes universitarios de asignaturas de contabilidad en la Universidad Pompeu Fabra después de modificar la metodología de enseñanza acercándose al Modelo de Aprendizaje. Los resultados indican un aumento de la satisfacción en el proceso de aprendizaje. Esta satisfacción fue mayor durante los años iniciales de "experimentación" que en los años de "consolidación" de esta metodología. La caída de la satisfacción coincide con una mayor carga de trabajo al incrementarse el número de asignaturas impartidas simultáneamente con esta metodología.

PALABRAS CLAVE: EEES, metodología de aprendizaje, evaluación continua, satisfacción estudiante

ABSTRACT: The adaptation of the European Higher Education Area (EHEA) in the Spanish universities it is implying a change of educational paradigm for undergraduate education. Traditionally, undergraduate education in Spain has been delivered under the Instruction Paradigm based on providing instruction. However, in the experimental introduction to the EEES a move towards the Learning Paradigm, the basis of which is to produce learning, has been observed.

This paper analyses the effects on students' satisfaction after modifying the teaching methodology so that subjects become nearer the Learning Paradigm. The data has been extracted from the satisfaction surveys completed by students in subjects belonging to the accounting area at Universitat Pompeu Fabra. The main results of the model show that the satisfaction was higher during the initial "testing" period of the methodology than in the

\section{*Ayudas:}

- Ayuda Generalitat de Catalunya: "Mejora de la calidad docente en las asignaturas de contabilidad". Investigador principal: Ester Oliveras. Referencia: 2007MQD00106

- Varias ayudas del CQUID (Centro para la calidad y la innovación docente) de la Universidad Pompeu Fabra

Artículo de investigación. Recibido: 27-07-10 - Versión revisada: 15-02-12, 11-01-13, Aceptado: 21-01-13

Licencia Creative Commons BY NC ND · 2013 · Asociación Española de Contabilidad y Administración de Empresas - AECA 
consolidation period. This satisfaction drop coincides with an increased student workload due to the higher number of subjects taught simultaneously under the new methodology.

KEYWORDS: EHEA, learning process, continuous assessment, student satisfaction

\section{INTRODUCCIÓN}

La primera década del siglo XXI se consolida como un punto de inflexión para el sistema educativo universitario. Las instituciones de educación superior han adaptado sus titulaciones para cumplir con los requisitos establecidos por el Espacio Europeo de Educación Superior (EEES). Aunque, actualmente, las nuevas titulaciones de Grado ya se ofrecen, este inicio ha sido precedido por un período de cinco o seis años durante el cual se han realizado experiencias aisladas con la nueva metodología docente. Este es el caso del área de contabilidad de la Universidad Pompeu Fabra.

En este artículo se mide el impacto en la satisfacción del estudiante al pasar de una metodología tradicional a una metodología más interactiva y centrada en el alumno durante un período de seis años, del 2003 al 2009, en que el número de asignaturas en fase experimental iba incrementando.

Iniciaremos el artículo con una revisión de la adopción del EEES, a continuación se realizará una revisión de la literatura y, finalmente, se muestran el método y los resultados del presente estudio.

\section{ANTECEDENTES: EL NUEVO MODELO EDUCATIVO Y DESARROLLO DEL EEES}

La Declaración de Bolonia en 1999, consensuada por los ministros de educación de 29 países, supuso el inicio del Espacio Europeo de Educación Superior que tendría su culminación diez años más tarde con la entrada en funcionamiento de las nuevas titulaciones universitarias: los Grados. Sin embargo, hay que reconocer el mérito de un encuentro anterior, realizado en 1988 por Italia, Alemania y el Reino Unido en La Sorbona en la que se firmó una Declaración que ya apuntaba a la convergencia gradual de los sistemas de educación superior en Europa.

Desde la declaración de Bolonia, y para el correcto avance de este proceso, se realizan conferencias con periodicidad bianual con los objetivos de revisar los avances realizados y establecer prioridades para el próximo bienio. Hasta el presente, las conferencias se han celebrado en Praga (2001), Berlín (2003), Bergen (2005), Londres (2007) y, la última de ellas, se realizó en Lovaina (2009).

El EEES establece criterios de convergencia europea y ofrece mecanismos que facilitan la adopción de títulos universitarios comparables. Los objetivos comunes aumentan el atractivo y la competitividad de las universidades europeas a nivel internacional. Los criterios o valores promovidos por el EEES son: el respeto por la diversidad cultural y educativa en Europa; aumentar la competitividad de la universidad europea en el ámbito internacional; promover la movilidad de profesionales y estudiantes; mejorar la calidad y la excelencia educativa.

Algunos estudios demuestran que la puesta en marcha del EEES está impactando más allá de las fronteras geográficas europeas (Brooks y Huisman, 2009; Zgaga, 2003). Por lo tanto, independientemente de la profundidad del cambio, es evidente que existe un interés mundial por la creación de un sistema internacional de educación que promueva la compatibilidad y la comparabilidad (Papatsiba, 2006). 


\section{MARCO TEÓRICO}

Naturalmente, el proceso de implantación del EEES está siendo analizado y discutido por la comunidad académica desde un punto de vista pedagógico. En el marco teórico, destaca la temprana aportación de Barr y Tagg (1995) que califican el nuevo modelo educativo como un cambio de paradigma, pasando del Paradigma Instructivo al Paradigma de Aprendizaje. Este cambio se inició primero en los Estados Unidos, para posteriormente introducirse en Europa. A nivel europeo, es habitual referirse a metodología Bolonia, como sinónimo de Paradigma de Aprendizaje en el contexto de integración en el EEES y es en ese sentido, que se usará en el presente trabajo.

Según los autores el Paradigma Instructivo se basa, principalmente, en una metodología de carácter expositivo. Bajo este paradigma, el estudiante tiene un papel pasivo en el proceso de aprendizaje. En contraposición, el nuevo paradigma sitúa al estudiante en el centro del proceso de aprendizaje. En la Tabla 1, se detallan las principales diferencias entre los dos paradigmas.

Tabla 1. Comparativa de los modelos educativos

\begin{tabular}{|c|c|c|}
\hline & Paradigma Instructivo & Paradigma de Aprendizaje \\
\hline MISIÓN & Proporcionar instrucción & Producir aprendizaje \\
\hline \multirow{3}{*}{$\begin{array}{l}\text { Estructuras de } \\
\text { ense Ñanza y } \\
\text { aprendizaje }\end{array}$} & Cubrir material & Resultados de aprendizaje especificados \\
\hline & Evaluación final & Evaluación inicial/continua/ final \\
\hline & $\begin{array}{l}\text { Grado equivale a la } \\
\text { acumulación de horas- } \\
\text { créditos }\end{array}$ & $\begin{array}{l}\text { Grado equivale a conocimientos y } \\
\text { habilidades demostradas }\end{array}$ \\
\hline \multirow[t]{3}{*}{$\begin{array}{l}\text { Teoría del } \\
\text { aprendizaje }\end{array}$} & $\begin{array}{l}\text { El conocimiento existe } \\
\text { "allí fuera" }\end{array}$ & $\begin{array}{l}\text { El conocimiento existe en la mente de } \\
\text { cada persona y está configurado según la } \\
\text { experiencia individual }\end{array}$ \\
\hline & $\begin{array}{l}\text { El aprendizaje es } \\
\text { acumulativo y lineal }\end{array}$ & $\begin{array}{l}\text { El aprendizaje es la creación e } \\
\text { interacción de marcos mentales }\end{array}$ \\
\hline & $\begin{array}{l}\text { Estudiante y profesor } \\
\text { deben estar presentes }\end{array}$ & $\begin{array}{l}\text { El aprendizaje está centrado y controlado } \\
\text { por el estudiante }\end{array}$ \\
\hline \multirow{2}{*}{$\begin{array}{l}\text { Naturaleza de los } \\
\text { roles }\end{array}$} & $\begin{array}{l}\text { La facultad son } \\
\text { principalmente los } \\
\text { profesores }\end{array}$ & $\begin{array}{l}\text { El profesorado es principalmente diseñador } \\
\text { de métodos y ambientes de aprendizaje }\end{array}$ \\
\hline & $\begin{array}{l}\text { Los profesores clasifican } \\
\text { y ordenan a los } \\
\text { estudiantes }\end{array}$ & $\begin{array}{l}\text { Todo el personal son educadores que } \\
\text { producen el aprendizaje y el éxito del } \\
\text { estudiante }\end{array}$ \\
\hline
\end{tabular}

Fuente: Adaptado de Barr y Tagg (1995)

Aunque este cambio legislativo está impulsado desde la Unión Europea, los Gobiernos nacionales son los encargados de traducirlo a través de legislaciones nacionales 0 autonómicas. Después corresponde a cada universidad en particular articularlo. En su 
última etapa, los actores finales de la implantación en las aulas son los profesores. Los estudiantes y la sociedad en general, serán los receptores de esta nueva metodología. Este proceso se muestra gráficamente en la Figura 1.

Figura 1. Del Paradigma de Instrucción al Paradigma de Educación PARADIGMA DE INSTRUCCIÓN

\section{Paradigma de Instrucción}

Unión Europea $\Rightarrow$ Estados $\Rightarrow$ Universidades $\Rightarrow$ Implantación en el aula $\Rightarrow$ Sociedad

Profesores - Estudiantes

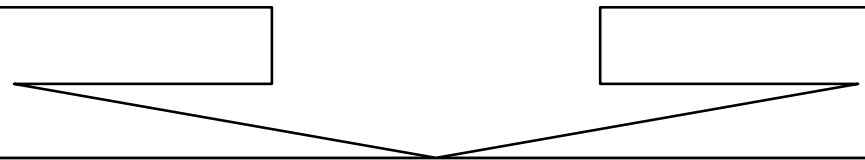

\section{Paradigma de Educación}

Se observa que se trata de un proceso de cuatro fases. Los estudios académicos realizados sobre el proceso de Bolonia pueden clasificarse teniendo en cuenta este esquema.

- Fase 1. Estudios entorno a la implantación en los estados miembros de la Unión Europea.

- Fase 2. Estudios entorno a la implantación en las universidades.

- Fase 3. Estudios sobre metodologías docentes y su impacto sobre el rendimiento y satisfacción de los estudiantes.

- Fase 4. Estudios sobre el impacto en la sociedad de este cambio.

A continuación comentamos algunos de los estudios más significativos en cada una de las fases.

En la fase 1, encontramos el estudio de González, Arquero y Hassall (2009) que compara las diferencias en la implantación entre el Reino Unido y España. En este estudio se constata que el Reino Unido gozaba de una legislación previa más cercana a los requisitos de Bolonia. Por lo que se deduce, que el cambio legislativo y cultural que supone para España este cambio de paradigma es más acusado.

En la fase 2 se englobarían los estudios que analizan como se han implantado los nuevos grados en las diferentes universidades. Por ejemplo, los criterios generales recomendados respecto a la importancia de la evaluación continua, el número de estudiantes por clase o el tipo de atención que se proporciona al estudiante. En esta línea, mencionar el estudio en 12 países de Vläscenau y Voicu (2006) que muestran que la adaptación es más rápida en las instituciones públicas que en las privadas.

En esta fase, se constatan algunos estudios críticos respecto al proceso de implantación en las universidades. Por ejemplo, el estudio de Veiga y Amaral (2009) es escéptico respecto a la calidad de la implantación del proceso de Bolonia en las universidades de Portugal. A nivel estatal, destaca el trabajo de Arquero, González y 
Hassall (2011) en el que constatan que algunos de los fundamentos de la metodología de la enseñanza enfocada en la transmisión de contenidos han permanecido en la configuración actual de la enseñanza por competencias. Pascual, Camacho, Urquía y Müller (2011), cuestionan la metodología de la enseñanza activa y su consecuente método de evaluación. La experiencia la aplican profesores de Contabilidad Financiera de primer curso de la universidad Complutense de Madrid. A partir de los resultados obtenidos ponen en duda el sistema de evaluación recogido en los planes de estudios aprobados por la Agencia Nacional de Evaluación de la Calidad y Acreditación (ANECA) para los estudios de GADE y GECO.

En la fase 3 se concentran una gran cantidad de trabajos académicos originados a partir de las experiencias de profesores al aplicar nuevos métodos de trabajo y el resultado obtenido por los estudiantes, en términos de satisfacción y de rendimiento académico. En términos de satisfacción de los estudiantes, la mayor parte de los estudios indican que una mayor satisfacción al aplicar metodologías participativas (Munn, y Gyffin, 1973, Clarke y Lane, 2005; Mingorance, 2008; Garandillas y otros, 2011). Sin embargo, existen resultados divergentes respecto a si estás metodologías conllevan unos mejores resultados académicos. Por ejemplo, en el estudio de Beltrán, Pereira y Sáez (2011) los resultados indican un mayor grado de satisfacción del alumnado ante estas nuevas técnicas docentes pero no unos mejores resultados académicos.

También existen estudios que analizan las características del estudiante, en particular, el estilo de aprendizaje, y las dificultades que puede comportar este sistema (Arquero y Tejero, 2006, 2011). En particular, Arquero y Tejero (2011) analizan la adaptación de los estudiantes de contabilidad ante las nuevas exigencias de Bolonia en España. Comparan el estilo de aprendizaje de estos estudiantes respecto a estudiantes de otras carreras cercanas dentro del ámbito de las ciencias sociales. La conclusión es que los estudiantes de contabilidad son mucho más dependientes en su estilo de aprendizaje que los otros. Esto hace que no se sientan cómodos con metodologías que les exijan autonomía en el aprendizaje en cuestión.

Por último, en la fase 4 se incluyen los estudios que analizan el impacto sobre la sociedad. En esta línea, comentar el artículo de Papatsiba (2006) que analiza si el proceso de convergencia incrementará la movilidad entre estudiantes.

El presente estudio se enmarca en la Fase 3.

\section{OBJETIVOS}

El objetivo de este trabajo es valorar el nivel de satisfacción de los estudiantes durante el período experimental de implantación de metodología Bolonia, entre el 2003-2009. También se valorará cómo evoluciona la satisfacción a medida que incrementa el número de asignaturas que se imparten con metodología Bolonia y si existen algunas asignaturas más aptas para ser impartidas con esta metodología. En este sentido, se parte del supuesto que asignaturas de segundo ciclo y con un grado alto de aplicabilidad son más aptas para un formato Bolonia.

En línea con estudios previos, se esperaba que el uso de metodología Bolonia incrementara de forma generalizada la satisfacción de los estudiantes. También, se anticipaba un incremento mayor en la satisfacción de la asignatura de Análisis de Estados Financieros, al tratarse de una asignatura de tercer curso que permite aplicar los conocimientos al análisis de una empresa real, en un trabajo en grupo. La metodología Bolonia parecía más apropiada en esta asignatura, que en otras asignaturas de carácter más introductorio. Por último, no existía ninguna evidencia que la satisfacción de los alumnos debería variar a lo largo del período estudiado, 
utilizando la misma metodología. Estos razonamientos, nos llevan a plantear las siguientes cuestiones:

Cuestión 1: La satisfacción del alumnado es mayor con la metodología de Bolonia.

Cuestión 2: Dadas las características de las asignaturas, podríamos pensar que la asignatura de Análisis de los Estados Financieros es más proclive a la metodología de Bolonia.

Cuestión 3: La satisfacción de los estudiantes se mantiene independientemente del año académico.

\section{MÉTODO}

Los datos han sido extraídos de las encuestas de satisfacción realizadas por los alumnos en tres asignaturas pertenecientes al área de contabilidad de la Universidad Pompeu Fabra en Barcelona: Introducción a la Contabilidad Financiera, Análisis de Estados Financieros e Introducción a la Contabilidad Analítica.

La experimentación con metodología Bolonia en la Universidad Pompeu Fabra, empezó en el año 2003 y se extendió hasta el 2009, fecha de implantación de los nuevos grado. Durante el período, 2003-2009, los profesores podían optar al plan experimental, de manera voluntaria. Básicamente, las asignaturas consideradas metodología Bolonia reúnen todas o algunas de las siguientes características:

- Plan docente con competencias definidas, así como los instrumentos de evaluación.

- Presencia de la evaluación continua (con un peso de entre 40-60\% de la nota final);

- Disminución del número de clases teóricas (entre un 30\% y un 50\%);

- Introducción de Seminarios en grupos reducidos.

Los datos cuantitativos se extraen de la encuesta institucional que se pasa a los estudiantes al finalizar cada asignatura. Las cuestiones utilizadas permiten valorar en una escala de 0 a 9 los siguientes aspectos:

- La satisfacción con la enseñanza recibida.

- El grado de cumplimiento con lo previsto en el programa de la asignatura.

- La adecuación del material didáctico.

Las variables explicativas utilizadas para el modelo cuantitativo:

- Curso académico: hay datos desde 2003/04 hasta 2008/09, dependiendo de la asignatura.

- Asignaturas:

- Introducción a la Contabilidad Financiera

- Introducción a la Contabilidad Analítica

- Análisis de los Estados Financieros

- Grupo: se refiere a los grupos que cursan cada una de las asignaturas antes mencionadas, en cada caso hay cuatro grupos: 1, 2, 3 y 4.

Se han creado tantas variables ficticias como categorías tiene cada variable. Bolonia: valor 1 si la asignatura sigue Bolonia y valor 0 en caso contrario. Conviene recordar que a efectos de evitar un problema de multicolinealidad perfecta se elimina de la 
estimación una de las categorías de cada una de las variables ficticias creadas. En la Tabla 2 se presenta un resumen de los datos analizados.

TABLA 2. Resumen de los datos analizados

\begin{tabular}{|c|c|c|c|}
\hline Asignaturas & Curso académico & Metodología Bolonia & Número de grupos \\
\hline $\begin{array}{c}\text { Introducción a la } \\
\text { Contabilidad } \\
\text { Financiera }\end{array}$ & $\begin{array}{c}\text { Desde } 2005 / 06 \text { hasta } \\
2008 / 09\end{array}$ & Desde 2007/08 & $\begin{array}{c}\text { Todos los grupos } \\
(1,2,3 \text { y } 4)\end{array}$ \\
\hline $\begin{array}{c}\text { Introducción a la } \\
\text { Contabilidad } \\
\text { Analítica }\end{array}$ & $\begin{array}{c}\text { Desde } 2003 / 04 \text { hasta } \\
2008 / 09\end{array}$ & Desde 2005/06 & $\begin{array}{c}\text { Todos los grupos } \\
(1,2,3 \text { y } 4)\end{array}$ \\
\hline $\begin{array}{c}\text { Análisis de los } \\
\text { Estados Financieros }\end{array}$ & $\begin{array}{c}\text { Desde 2003/04 hasta } \\
2008 / 09\end{array}$ & Desde 2004/05 & $\begin{array}{c}\text { Sólo uno de los cuatro } \\
\text { grupos }\end{array}$ \\
\hline
\end{tabular}

La satisfacción de los estudiantes se analiza a partir de dos cursos académicos antes de la introducción de la metodología de Bolonia.

Hay cuatro grupos de estudiantes que se imparten simultáneamente en cada asignatura. Cada grupo tienen entre 80 y 100 alumnos matriculados. En algunas asignaturas o cursos académicos, no se aplicaba la metodología de Bolonia a todos los grupos.

Se dispone de datos sobre la satisfacción desde el curso académico 2005/06 al 2008/09 para una asignatura y desde el 2003/04 hasta el 2008/09 para el resto. En la Tabla 2 se informa sobre desde cuando se ha procedido a aplicar la "experimentación" de Bolonia, en cada caso. En todas las asignaturas, con excepción de Análisis de los Estados Financieros, cuando se aplica la metodología de Bolonia se ha hecho en los 4 grupos en que se divide cada asignatura. En el caso de Análisis de los Estados Financieros, sólo el grupo 1 ha experimentado la nueva metodología, el resto de grupos han seguido la metodología tradicional.

Además de los datos cuantitativos, el estudio se ha complementado, con las observaciones de los estudiantes en preguntas de formato abierto. En estos espacios, los estudiantes comentan libremente sus opiniones en relación al profesor, metodología, propuestas de mejoras o cualquier otro aspecto que consideren relevante.

\section{RESULTADOS}

En la Tabla 3 se presenta información cuantitativa acerca de la media de la satisfacción global del alumno en función de si la asignatura ha sido impartida utilizando la metodología de Bolonia o no. En este sentido, se observa, en primer lugar como, en el caso de que la asignatura haya sido impartida siguiendo la metodología de Bolonia la satisfacción global media es mayor $(7,66)$ que en el caso contrario $(7,03)$. Sin embargo, en la tercera columna de la Tabla 3 , se observa como estas diferencias no llegan a ser estadísticamente significativas al $5 \%$.

\footnotetext{
${ }^{1}$ Estas diferencias sí se pueden considerar significativamente distintas bajando el nivel al $10 \%$.
} 
TABLA 3. TEST dE COMPARACIÓN DE LAS SATISFACCIONES MEDIAS SEGÚN LA METODOLOGÍA EN LA QUE HAN RECIBIDO LOS CURSOS.

\begin{tabular}{|l|c|c|l|}
\hline \multicolumn{1}{|c|}{ Variable } & Bolonia & No Bolonia & $\begin{array}{c}\text { Hipótesis igualdad (5\% } \\
\text { significación) }\end{array}$ \\
\hline Satisfacción global & 7,66 & 7,03 & $\begin{array}{l}\dagger=|-1,99|<2,01 \\
\text { No Rechazada }\end{array}$ \\
\hline Material didáctico adecuado & 7.64 & 7 & $\begin{array}{l}\dagger=|-1.98|<2.01 \\
\text { No Rechazada }\end{array}$ \\
\hline $\begin{array}{l}\text { Se cumple con el programa } \\
\text { de la asignatura }\end{array}$ & 8.28 & 7.55 & $\begin{array}{l}\dagger=|-1.95|<2.01 \\
\text { No Rechazada }\end{array}$ \\
\hline
\end{tabular}

La anterior evidencia no tiene en cuenta qué otros factores afectan a la satisfacción global media del estudiante, por lo que las anteriores cifras puedan estar recogiendo indirectamente el efecto de otras variables que a lo largo de los cursos analizados también hayan cambiado, como ejemplo, el curso, la asignatura o el grupo analizados.

A fin de tener en cuenta dichos factores, y medir adecuadamente cómo ha variado la satisfacción global media del alumno con unas características dadas, se ha procedido a estimar un modelo explicativo de dicha variable.

La especificación econométrica de dicho modelo es:

Sat.media $_{i}=\beta+\alpha_{i} \sum_{i=1}^{i=6}$ Curso $_{i}+\delta_{k} \sum_{k=1}^{k=3}$ Asignatura $+\gamma_{l} \sum_{l=1}^{l=4}$ grupo $+\tau B o \log n a+u_{i}$

Donde $\alpha, \beta, \delta, \gamma \quad y \tau$ son parámetros a estimar.

En la Tabla 4 se presentan los principales resultados de la estimación del modelo². Procediendo al comentario de los coeficientes, observamos como el grupo al cual pertenecen los estudiantes no aparece como una variable estadísticamente significativa.

\footnotetext{
2 El modelo se ha estimado por Mínimos Cuadrados Ordinarios.
}

En términos generales, la bondad del ajuste del modelo $(0,37,0,35$ y 0,38 para las distintas variables a explicar) se puede considerar correcta, teniendo en cuenta que se trata de un modelo con datos individuales. El valor del estadístico $\mathrm{F}$ del contraste de significación conjunta $(2,56)$ nos permite rechazar, al $5 \%$ del nivel de significación, la hipótesis nula de que todas las variables del modelo resultan no significativas. 
TABla 4. Estimación del MOdelo eXPlicativo de la SATISfaCción GLOBAL MEDIA DEL ESTUDIANTE.

\begin{tabular}{|c|c|c|c|}
\hline Variable & $\begin{array}{l}\text { Satisfacción } \\
\text { general }\end{array}$ & $\begin{array}{c}\text { El material didáctico es } \\
\text { adecuado }\end{array}$ & $\begin{array}{l}\text { Se hace lo que } \\
\text { prevé el programa } \\
\text { de la asignatura }\end{array}$ \\
\hline \multicolumn{4}{|l|}{ Curso(ref:2003/2004) } \\
\hline $2004 / 2005$ & $-0.95^{*}$ & -0.83 & -0.70 \\
\hline $2005 / 2006$ & $-0.97^{*}$ & -0.80 & -0.74 \\
\hline $2006 / 2007$ & $-0.96^{*}$ & $-0.80^{*}$ & $-0.78^{*}$ \\
\hline $2007 / 2008$ & $-1.40^{* *}$ & $-1.07^{*}$ & $-1.07^{*}$ \\
\hline $2008 / 2009$ & $-1.36^{* *}$ & $-1.07^{*}$ & $-1.05^{*}$ \\
\hline \multicolumn{4}{|l|}{$\begin{array}{l}\text { Asignatura (ref: Análisis de } \\
\text { los Estados Financieros) }\end{array}$} \\
\hline $\begin{array}{l}\text { Introducción a la } \\
\text { Contabilidad Financiera }\end{array}$ & $1.10^{* *}$ & $-1.46^{* *}$ & $-1.17^{* *}$ \\
\hline $\begin{array}{l}\text { Introducción a la } \\
\text { Contabilidad Analítica }\end{array}$ & -0.49 & $-0.41^{* *}$ & $-1.05^{* *}$ \\
\hline \multicolumn{4}{|l|}{ Grupo (ref:1) } \\
\hline 2 & 0.09 & -0.08 & 0.62 \\
\hline 3 & -0.52 & -0.49 & -0.61 \\
\hline 4 & -0.24 & -0.22 & -0.26 \\
\hline Bolonia & $1.17^{* *}$ & $1.10^{* *}$ & $1.05^{* *}$ \\
\hline Constante & $7.82^{* *}$ & $8.63^{* *}$ & $9.14^{* *}$ \\
\hline$N=59$ & $R^{2}=0.37$ & $R^{2}=0.35$ & $R^{2}=0.38$ \\
\hline
\end{tabular}

** variables estadísticamente significativas al $5 \%$ de nivel de significación.

* variables estadísticamente significativas al 10\% de nivel de significación.

En cuanto a la variable curso, todos los cursos aparecen como estadísticamente significativos al $10 \%$ del nivel de significación (satisfacción de los estudiantes en general) y cursos 2006/2007, 2007/2008 y 2008/2009 (variable "el material didáctico es adecuado" y variable "se hace lo que prevé el programa de la asignatura"). En todos los casos, respecto a la categoría de referencia (curso 2003/2004), en todos los cursos se aprecia un descenso del nivel de la satisfacción global media que oscila entre los 0,95 puntos del curso 2004/2005 a los 1,40 del curso 2007/2008 (satisfacción de los estudiantes en general), entre los 0,80 puntos en el año 2006/2007 a los 1,07 en el año 
$2007 / 2008$ y $2008 / 2009$ (variable "el material didáctico es adecuado") y entre 0,78 puntos en el año 2006/2007 a los 1,07 en el año 2007/2008 (variable "se hace lo que prevé el programa de la asignatura").

En cuanto a la asignatura, que el curso sea sobre "Introducción a la Contabilidad Financiera" aumenta en 1,10 puntos la satisfacción global media del estudiante, en relación a que el curso sea "Análisis de los Estados Financieros". Entre las asignaturas "Introducción a la Contabilidad Analítica" y "Análisis de los Estados Financieros" no se observan diferencias estadísticamente significativas en cuanto a la satisfacción de los estudiantes en general). Sin embargo, "Introducción a la Contabilidad Financiera" (por 1,46 y 1,17) y "Introducción a la Contabilidad Analítica (por 0,41 y 1,05 ) disminuye la satisfacción del estudiante en las variables "el material didáctico es adecuado" y "se hace lo que prevé el programa de la asignatura" en relación al curso "Análisis de los estados Financieros".

Finalmente, nuestra principal variable de interés, Bolonia, presenta un coeficiente positivo y estadísticamente significativo. Así, descontando el efecto del curso, asignatura y grupo analizado, el efecto propio de que la asignatura se haya impartido siguiendo la metodología docente de Bolonia aumenta la satisfacción global media del estudiante en 1,17 puntos. En términos de las variables "el material didáctico es adecuado" y "se hace lo que prevé el programa de la asignatura" seguir la metodología de Bolonia incrementa estas variables en 1,10 y 1,05 puntos respectivamente.

Retornando a las cuestiones planteadas la inicio de este apartado:

Cuestión 1: La satisfacción del alumnado es mayor con la metodología de Bolonia.

Esta cuestión ha sido confirmada, la satisfacción de los estudiantes aumenta con la introducción de la metodología Bolonia y, durante el período estudiado, se mantiene por encima de la satisfacción registrada los años previos a la introducción de la nueva metodología.

Cuestión 2: Dadas las características de las asignaturas, podríamos pensar que la asignatura de Análisis de los Estados Financieros es más proclive a la metodología de Bolonia.

La asignatura de Análisis de los Estados Financieros no registra un mayor nivel de satisfacción. Esto nos hace deducir que tenemos una preconcepción errónea de la adecuación de la metodología Bolonia para determinadas asignaturas.

El éxito del uso de metodología Bolonia en asignaturas de Introducción a la Contabilidad Financiera y de Introducción a la Contabilidad Analítica, indican que existen suficientes métodos de evaluación para asignaturas introductorias y para asignaturas más aplicadas.

Cuestión 3: La satisfacción de los estudiantes se mantiene independientemente del año académico.

Se comprueba que el nivel de satisfacción no se mantiene, sino que muestra una progresiva disminución en el nivel medio de satisfacción general. Esto puede ser debido a que, con el paso del tiempo se fue incrementando el número de asignaturas que introducían experimentación con metodología Bolonia y los estudiantes se han visto abrumados por el incremento de trabajo que supone la evaluación continua. Según los registros de la universidad, durante el curso académico 2005-06, había siete asignaturas adaptadas a la nueva metodología, en 2006-07, nueve y en 2007-2008 ya eran catorce; haciéndose evidente la necesidad de coordinación entre asignaturas impartidas simultáneamente. 
Es especialmente ilustrativo uno de los comentarios de estudiantes de una asignatura adaptada a Bolonia el curso 2006-07 (primer trimestre): "Mi comentario no es exclusivo de la asignatura, pero considero que también afecta en esta materia. Mi observación tiene relación con la carga de trabajo de los alumnos en las diversas asignaturas que estamos cursando. Considero que el volumen de materia y el total de tareas, actividades, trabajos, ejercicios... es excesivo. Sobre todo me refiero a la cantidad de conocimientos que se espera que adquiramos. Creo que no es efectivo".

Otro comentario en la misma línea, también del curso 2006-07 (segundo trimestre): "Bolonia no funciona, y no sé porque la gente no se da cuenta. Hay un problema de aprendizaje, con el sistema tradicional todo quedaba más claro, con los apuntes de clase y los manuales recomendados estábamos más preparados. Ahora tienes que dedicar todo el tiempo que tienes para hacer el trabajo en grupo y ya no tienes tiempo para estudiar. Cuando Bolonia se haga en todas las asignaturas será imposible, no se pueden llevar 5 materias con trabajos semanales en grupo."

Estas afirmaciones de los estudiantes son recurrentes a medida que el número de asignaturas impartidas con metodología Bolonia asciende, incluso antes de la implantación definitiva, en el curso 2009-10.

\section{CONCLUSIONES}

De los resultados obtenidos, podemos concluir que, en relación a las asignaturas de contabilidad, el cambio hacia un modelo basado en el aprendizaje ha aumentado la satisfacción general de los estudiantes en el proceso de "experimentación". Esto está en consonancia con estudios previos, según los cuales, la implicación del alumno es superior $y$, en la realización de la evaluación continua el estudiante recibe feedback sobre su evolución que le permite reaccionar y aumentar la dedicación a la asignatura, si es necesario.

Sin embargo, es importante destacar que eliminando el efecto de Bolonia, hay una paulatina disminución de la satisfacción de los estudiantes. La explicación es que a medida que ha ido avanzando el proceso de extensión del modelo basado en el aprendizaje a un mayor número de asignaturas, se ha producido una falta de coordinación entre las materias que se imparten simultáneamente, lo que ha podido conducir a una sobrecarga de trabajo para los estudiantes en algunos periodos. También puede jugar un papel importante la dilución del efecto "novedad" y el hecho de que los estudiantes de las materias de contabilidad son mucho más dependientes en su estilo de aprendizaje que los de otras carreras cercanas (Arquero y Tejero, 2011). Esto hace que no se sientan cómodos con metodologías que les exijan autonomía en su aprendizaje.

De hecho, en algunas universidades ha ido apareciendo la figura del coordinador cuya función es la de asegurase que no coincida un volumen excesivo de trabajo en un período de tiempo determinado, por ejemplo, que no haya 5 trabajos en equipo en las cinco asignaturas que se impartan al mismo tiempo. El cambio cultural que ha supuesto para los estudiantes adaptarse a una metodología más centrada en el estudiante choca ahora con un necesario cambio cultural por parte de la comunidad de profesores universitarios, al requerir una mayor coordinación entre profesores de la misma área y de otras áreas. 


\section{BIBLIOGRAFÍA}

ARquero, J.L., GONZÁLEZ, J.Ma y HASSALL, T. [2011]: El cambio hacia la metodología de la enseñanza basada en las competencias en la universidad española: el estudio de un caso. Congreso AECA, Granada.

ARQUERO, J.L. Y TEJERO, C. [2006]: Estilos de aprendizaje y tolerancia a la ambigüedad de los estudiantes universitarios. Diagnóstico y Reflexiones ante el EEES. Revista de Enseñanza Universitaria, Vol. 28: 7-17.

ARQUERO, J.L. Y TEJERO, C. [2011]: How well adapted are accounting students for Bologna? A comparative analysis of learning styles of Spanish social sciences students, EDUCADE, n²: 145-156.

BARR, R. y TAGG, J. [1995]: A new paradigm for Undergraduate Education, Change: The magazine of Higher Learning. Noviembre/Diciembre.

Beltrán, J.L., PereiRA, J.M. y SÁez OCEjo, J.L. [2011]: Aplicación práctica de técnicas docents para Contabilidad Financiera, EDUCADE, $n^{\circ} 2$ : 3-34.

BROOKS, M. y HuISMAN, J. [2009]: The Eagle and the Circle of Gold Stars: Does the Bolonia process affect US Higher Education?, Higher Education in Europe, vol. 34, núm. 1, abril: 3-23.

Clarke, A. y LANE, A. [2005]: Seminar and tutorial sessions: a case study evaluating relationships with academic performance and student satisfaction, Journal of Further and Higher Education vol. 29, núm. 1, febrero: 15-23.

GARANDILLAS, M., SOMOHANO, F.M. y MARTíNEZ, F.J. [2011]: Innovación y evaluación en el marco del Espacio Europeo de Educación Superior: Una experiencia piloto utilizando herramientas Web 2.0, EDUCADE, $n^{\circ} 2:$ : 85-111.

GonzÁlez, J.M., ARQUero, J.L. y HASSALL, T. [2009]: Bolonia and Beyond: A Comparative Study Focused on UK and Spanish Accounting Education, Higher Education in Europe, vol. 34, núm. 1: 113-125.

MingoranCE ARNÁlz, A.C. [2008]: Análisis comparado entre los resultados de una evaluación continua y otra puntual. El caso de la asignatura de Macroeconomía, Revista de Investigación Educativa, vol. 26, núm. 1, primer semestre 2008: 95-120.

MUNN, H.E. y GIFFIN, K. [1973]: Relationship between teachers' task-oriented behavior, interpersonal maintenance behaviour, student achievement, and student satisfaction, Communication Education, 22:4, 304-309.

PAPATSIBA, V. [2006]: Making higher education more European through student mobility? Revisiting EU initiatives in the context of the Bologna Process. Comparative Education, Vol. 42, Issue 1: 93-11.

Pascual, D., CAMACho, Ma M, URQUía, E. Y MÜller, A. [2011]: ¿̇on los nuevos criterios de evaluación en el marco del EEES adecuados para valorar el rendimiento academic de los alumnos? Experiencia en Contabilidad Financiera, EDUCADE, $n^{\circ} 2$ : 67-83.

VEIGA, A. Y AMARAL, A. [2009]: Survey on the implementation of the Bolonia process in Portugal, Higher Education, vol. 57, núm. 1, enero: 57-69.

VLÄSCEANU, L. Y VOICU, B. [2006]: Implementation of the Bolonia Objectives in a Sample of European Private Higher Education Institutions: Outcomes of a Survey, Higher Education in Europe, vol. 31, núm. 1, abril: 25-52.

ZGaGA, P. [2003]: The External dimension of the Bolonia process: Higher Education in South East Europe and the European Higher Education Area in a Global World, Higher Education in Europe, vol. 28, núm. 3, octubre: 251-258. 